\title{
A 2cM genome-wide scan of European Holstein cattle affected by classical BSE
}

\author{
Brenda M Murdoch ${ }^{1}$, Michael L Clawson², William W Laegreid ${ }^{3}$, Paul Stothard', Matthew Settles ${ }^{4}$, \\ Stephanie McKay ${ }^{1,5}$, Aparna Prasad ${ }^{1}$, Zhiquan Wang ${ }^{1}$, Stephen S Moore ${ }^{1 *}$, John L Williams ${ }^{6}$
}

\begin{abstract}
Background: Classical bovine spongiform encephalopathy (BSE) is an acquired prion disease that is invariably fatal in cattle and has been implicated as a significant human health risk. Polymorphisms that alter the prion protein of sheep or humans have been associated with variations in transmissible spongiform encephalopathy susceptibility or resistance. In contrast, there is no strong evidence that non-synonymous mutations in the bovine prion gene (PRNP) are associated with classical BSE disease susceptibility. However, two bovine PRNP insertion/deletion polymorphisms, one within the promoter region and the other in intron 1, have been associated with susceptibility to classical BSE. These associations do not explain the full extent of BSE susceptibility, and loci outside of PRNP appear to be associated with disease incidence in some cattle populations. To test for associations with BSE susceptibility, we conducted a genome wide scan using a panel of 3,072 single nucleotide polymorphism (SNP) markers on 814 animals representing cases and control Holstein cattle from the United Kingdom BSE epidemic.

Results: Two sets of BSE affected Holstein cattle were analyzed in this study, one set with known family relationships and the second set of paired cases with controls. The family set comprises half-sibling progeny from six sires. The progeny from four of these sires had previously been scanned with microsatellite markers. The results obtained from the current analysis of the family set yielded both some supporting and new results compared with those obtained in the earlier study. The results revealed 27 SNPs representing 18 chromosomes associated with incidence of BSE disease. These results confirm a region previously reported on chromosome 20, and identify additional regions on chromosomes 2, 14, 16, 21 and 28. This study did not identify a significant association near the PRNP in the family sample set. The only association found in the PRNP region was in the case-control sample set and this was not significant after multiple test correction. The genome scan of the case-control animals did not identify any associations that passed a stringent genome-wide significance threshold.

Conclusions: Several regions of the genome are statistically associated with the incidence of classical BSE in European Holstein cattle. Further investigation of loci on chromosomes 2, 14, 16, 20, 21 and 28 will be required to uncover any biological significance underlying these marker associations.
\end{abstract}

\section{Background}

Transmissible spongiform encephalopathies (TSEs) are fatal neurodegenerative diseases that have been identified in a number of mammalian species (humans, cattle, sheep, mice, etc.) [1]. One of the common characteristics of these diseases is the accumulation of abnormally folded prion protein within the central nervous system. The prion protein is a glycosyl-phosphatidylinositol $(\mathrm{GPI})$ anchored protein that has a native form $\left(\mathrm{PrP}^{\mathrm{c}}\right)$ for

\footnotetext{
* Correspondence: stephen.moore@ales.ualberta.ca
'Department of Agricultural, Food and Nutritional Science, University of

* Correspondence: stephen.moore@ales.ualberta.ca
'Department of Agricultural, Food and Nutritional Science, University of Alberta, Edmonton, Canada
}

which the secondary structure consists mainly of alphahelices. The disease-associated or misfolded form, $\operatorname{PrP}^{\text {Res }}$, has a substantial increase in the beta-pleated sheets content and reduction of the alpha-helices in comparison to the native form [2]. This altered confirmation is associated with an increased resistance to digestion with proteinase $\mathrm{K}$ [3]. Furthermore, the presence of $\operatorname{PrP}^{\text {Res }}$ behaves like a seed that promotes the conversion of further native $\operatorname{PrP}^{\mathrm{c}}$ to $\operatorname{PrP}^{\mathrm{Res}}$ via a mechanism that is to date not completely understood [4].

Specific PRNP alleles of non-synonymous polymorphisms in humans and sheep are associated with acquired 
TSE susceptibility [5-8]. This is not the case in cattle, although the deletion alleles of a 23 base pair insertion/ deletion (InDel) polymorphism in the bovine PRNP promoter region and of a 12 base pair InDel within intron 1 have been associated with incidence of classical bovine spongiform encephalopathy (BSE) [9-11]. Both of these polymorphisms are contained in a block of high linkage disequilibrium (LD) within PRNP that appears conserved in many cattle populations, and is entirely outside of the coding region [12]. Thus, the full extent of PRNP association with classical BSE is currently not known. Previous genetic studies of BSE cattle have identified putative loci other than PRNP (located on chromosome 13 at $47.2 \mathrm{Mb}$ ) that are associated with incidence of disease $[13,14]$. These studies carried out low density whole-genome scans with microsatellite markers approximately every $20 \mathrm{cM}$ in female European Holstein cattle which contracted BSE and unaffected half-sib controls.

The objective of this study was to test loci throughout the bovine genome for an association with classical BSE using markers at $2 \mathrm{cM}$ resolution. This resolution is approximately a ten-fold improvement over previous genome scans of BSE samples. Two animal sample sets were used, allowing for two analysis approaches: a casecontrol association study and family based sib-transmission disequilibrium test (sib-TDT) study [13,14]. In both sample sets the cattle used were female European Holstein cattle which contracted BSE in the late 1980's and early 1990's (family analysis) and in the mid-1990's (case-control analysis) and were identified on commercial farms where the most likely source of disease was through consumption of contaminated feed during the United Kingdom's BSE epidemic. Although the United Kingdom imposed a ruminant feed ban of meat and bone meal in June 1988, it wasn't until August 1996 that a total ban on bovine meat and bone meal was implemented [15].

\section{Results}

The panel of SNP markers used in this study consisted of 3,072 SNPs dispersed across the genome at an approximate average interval of $2 \mathrm{cM}$ [16]. Of these, an average 2,853 passed quality control measures and had an average genotyping success rate of 0.99 . The samples used in this study came from two sets of animals with different relatedness that allowed for the use of several statistical analyses to test the association between SNP genotypes with classical BSE incidence. By using both offspring from six sires (sib-TDT) and (case-control) Holstein animals, this study examined within-breed and within-family SNP association with disease incidence.

\section{Family based association testing for BSE incidence}

The related sample set $(\mathrm{N}=481)$ was comprised of six paternal half-sib sire families of which 4 were scanned previously with microsatellites $[13,14]$. Samples were not available from either the sires or the dams; therefore, the sib-TDT analysis method [17] was used. Additionally, PLINK software [18] was used to establish an empirical $\mathrm{p}$ value and determine significance. The results for this sib-TDT analysis (412 animals across 2,827 SNPs after quality control) identified 46 SNPs that passed the Bonferroni correction with a $\mathrm{p}<0.05$ and 27 SNPs that passed the Bonferroni correction with a p < 0.01 (Table 1). In addition, a 10,000,000 permutation test was performed on this data using the PLINK "max $T$ ” to establish genome wide empirical $p$ values. The genome wide 10,000,000 permutation identified 31 SNPs with an empirical permutation of $\mathrm{p}<0.01$. This group of SNPs included the 27 SNPs which passed the Bonferroni at $\mathrm{p}<0.01$ plus four additional SNPs. Many of these SNPs are located in very close proximity [16] to one another and are potentially in LD in the population.

\section{Case-control association testing for BSE incidence}

The case-control samples were comprised of 149 BSE case and 184 control animals. The control samples include a least one animal collected from the same farm as each of the BSE cases as well as the controls for, and 15 BSE negative animals. The genotyping data on these animals was analyzed for an association with disease status using the case-control allelic test within the PLINK software [18]. This analysis (320 animal across 2,872 SNPs after quality control) revealed 20 SNPs with a $\mathrm{p}<0.01,14$ SNPs with a $\mathrm{p}<0.005$ and 6 SNPs with a $\mathrm{p}<0.001$. In order to determine the number of these SNPs that may have occurred by chance, an empirical pvalue for each single SNP and across all SNPs (genome wide) was calculated using the $\max (\mathrm{T})$ permutation procedure with 10,000 permutations. Following correction for the false discovery rate no significant associations at $\mathrm{p}$ $\leq 0.05$ genome-wide significance were identified in this data set. This was consistent with the use of Bonferroni multi-test correction on this data set, where again none of the SNPs achieved significance of $\mathrm{p} \leq 0.05$. To assess significance a threshold was set at $\mathrm{p} \leq 1.7 \times 10^{-5}$ (Bonferroni calculation). Twenty SNPs were identified with $\mathrm{p}<0.01$ (Table 2), where four SNPs had a $\mathrm{p} \leq 5 \times 10^{-4}$ and one SNP had a $\mathrm{p} \leq 1 \times 10^{-4}$. Thus a single SNP had a suggestive association with BSE incidence on chromosome $14\left(\mathrm{p}=7.25 \times 10^{-5}\right)$. SNPs which did not quite reach this threshold but had a $\mathrm{p} \leq 5 \times 10^{-4}$ were found on chromosomes $4,10,14$, and 15 . The number of loci on each chromosome, the identity of these specific SNPs and their corresponding p-values are reported in Table 2. 
Table 1 The results of sib-TDT model analysis using the large family sample set.

\begin{tabular}{|c|c|c|c|c|c|c|}
\hline SNP ID & CHR & $\begin{array}{l}\text { Location } \\
\text { bp }\end{array}$ & Gene & $\begin{array}{c}\text { unadjusted } \\
\mathrm{p} \text { value }\end{array}$ & $\begin{array}{c}\text { permuted } \\
\mathrm{p} \text { value }\end{array}$ & Bonferroni \\
\hline AAFC02065030 & 2 & $37,055,124$ & & $1.93 \mathrm{E}-08$ & $3.61 \mathrm{E}-05$ & $5.45 \mathrm{E}-05$ \\
\hline rs29020694 & 4 & $90,418,076$ & & $1.87 \mathrm{E}-06$ & 4.05E-03 & $5.29 \mathrm{E}-03$ \\
\hline AAFC02132123_1 & 4 & $117,126,683$ & LOC100138299 & $9.38 \mathrm{E}-07$ & $1.98 \mathrm{E}-03$ & 2.65E-03 \\
\hline AAFC02132123_2 & 4 & $117,126,810$ & LOC100138299 & $3.38 \mathrm{E}-06$ & 7.33E-03 & $9.55 \mathrm{E}-03$ \\
\hline AAFC02012009 & 5 & $30,554,866$ & LOC507184 & $3.53 \mathrm{E}-06$ & 7.69E-03 & $9.98 \mathrm{E}-03$ \\
\hline rs29003193 & 5 & $66,501,993$ & & $3.12 \mathrm{E}-08$ & 5.79E-05 & $8.82 \mathrm{E}-05$ \\
\hline rs29012226 & 5 & $67,701,052$ & ANKS1B & $6.63 \mathrm{E}-07$ & $1.38 \mathrm{E}-03$ & 1.87E-03 \\
\hline SCAFFOLD106936 & 6 & $98,767,642$ & & $6.35 \mathrm{E}-07$ & $1.32 \mathrm{E}-03$ & 1.80E-03 \\
\hline rs29016161 & 7 & $24,604,791$ & & $2.45 \mathrm{E}-06$ & 5.31E-03 & $6.93 \mathrm{E}-03$ \\
\hline rs29017305 & 9 & $62,946,258$ & $\mathrm{BACH} 2$ & $2.30 \mathrm{E}-06$ & 4.97E-03 & $6.49 \mathrm{E}-03$ \\
\hline rs29013631 & 10 & $38,725,566$ & & $2.25 \mathrm{E}-06$ & 4.87E-03 & $6.36 \mathrm{E}-03$ \\
\hline rs29022366 & 12 & $80,076,729$ & LOC786668 & $2.74 \mathrm{E}-06$ & 5.95E-03 & 7.75E-03 \\
\hline rs29010388 & 14 & $4,145,186$ & & $4.20 \mathrm{E}-07$ & $8.56 \mathrm{E}-04$ & 1.19E-03 \\
\hline AAFC02138417 & 15 & $70,632,844$ & & 3.31E-06 & 7.19E-03 & $9.36 \mathrm{E}-03$ \\
\hline rs29010371 & 16 & $63,558,950$ & FAM129A & 1.06E-06 & $2.26 \mathrm{E}-03$ & $3.00 \mathrm{E}-03$ \\
\hline rs29009572 & $17^{\#}$ & $37,575,810$ & & $1.29 \mathrm{E}-06$ & $2.76 \mathrm{E}-03$ & $3.65 \mathrm{E}-03$ \\
\hline rs29021871 & 17 & $44,213,230$ & & $1.06 \mathrm{E}-06$ & $2.25 \mathrm{E}-03$ & 2.99E-03 \\
\hline CART & $20^{\#}$ & $4,922,252$ & CART & $3.57 \mathrm{E}-08$ & $6.75 \mathrm{E}-05$ & $1.01 \mathrm{E}-04$ \\
\hline rs29018531 & 20 & $38,814,738$ & & $1.32 \mathrm{E}-06$ & $2.82 \mathrm{E}-03$ & $3.72 \mathrm{E}-03$ \\
\hline AAFC02028192 & 21 & $5,702,403$ & MEF2A & $1.24 \mathrm{E}-07$ & $2.49 \mathrm{E}-04$ & $3.49 \mathrm{E}-04$ \\
\hline rs29022862 & 21 & $12,595,937$ & & $1.85 \mathrm{E}-07$ & $3.73 \mathrm{E}-04$ & $5.22 \mathrm{E}-04$ \\
\hline rs29009825 & 21 & $24,827,395$ & & $1.90 \mathrm{E}-06$ & 4.11E-03 & $5.38 \mathrm{E}-03$ \\
\hline rs29012664 & 22 & $42,415,179$ & FHIT & $5.30 \mathrm{E}-07$ & $1.10 \mathrm{E}-03$ & $1.50 \mathrm{E}-03$ \\
\hline NW_930303_1 & $24^{\#}$ & $29,286,364$ & & $1.02 \mathrm{E}-07$ & 2.05E-04 & $2.90 \mathrm{E}-04$ \\
\hline NW_930303_2 & $24^{\#}$ & $29,286,314$ & & 3.06E-07 & $6.17 \mathrm{E}-04$ & 8.64E-04 \\
\hline SCAFFOLD176855 & 28 & $26,871,628$ & SLC29A3 & $1.03 \mathrm{E}-06$ & $2.18 \mathrm{E}-03$ & 2.90E-03 \\
\hline SCAFFOLD68962 & $x$ & $7,641,750$ & & 3.15E-06 & $6.86 \mathrm{E}-03$ & 8.91E-03 \\
\hline
\end{tabular}

Locations were determined by blast to bovine sequence version 4.0. All other locations denoted by ${ }^{\text {\# }}$ were either determined from bovine sequence version 2.0 , 4.1 or the Maryland sequence assembly. Permuted $p$ values reported here are from a 10,000,000 genome-wide SNP permutation.

This data set was also subjected to a best fit model test where the standard allelic, trend, dominance, recessive and genotypic association tests were performed, and the test with the lowest $\mathrm{p}$ value was reported. All of the SNPs identified above were also identified in the best fit model as either allelic or trend, however additional SNPs with recessive, dominant and genotype associations were also identified. In the best fit model there were a total of fourteen SNPs with a recessive mode of action, seven dominant SNPs and eight genotypic SNPs with a $\mathrm{p}<$ 0.01 (see Additional file 1). Using the same thresholds as described above, (i.e. significant with a $\mathrm{p} \leq 10^{-5}$ and suggestive with a $\left.\mathrm{p} \leq 10^{-4}\right)$, one SNP on chromosome 14 had a suggestive association with BSE incidence.

\section{Discussion}

\section{Classical BSE as a phenotype}

Clinical presentation of BSE disease is a difficult phenotype to test for genetic associations. Animals that have developed BSE are clearly susceptible, however, those which are clinically healthy are difficult to assess. In the present study, clinically healthy animals were used as controls, however, these animals may have been incubating disease or may not have ingested enough infectious agent to become symptomatic, or alternatively, they may in fact have been resistant to disease. Therefore, the analysis was performed with the realization that phenotypic noise in the controls will have reduced the power to detect associations. Another consideration is that classical BSE is a complex trait which may be more consistent with interactive and possibly subtle effects of multiple contributing loci. Therefore, multiple testing corrections applied to results such as these may be overly prone to type II errors (i.e. discarding real associations). Consequently, it is important to examine the results for supporting evidence of associations between disease and genetic loci, as discussed below.

\section{PRNP gene}

PRNP variation was not exhaustively tested for an association with classical BSE as the focus of this study was primarily genome wide. Over 380 polymorphisms are 
Table 2 PLINK case-control association results of the case-control sample set.

\begin{tabular}{lcccc}
\hline SNP ID & CHR & $\begin{array}{c}\text { Location } \\
\text { bp }\end{array}$ & Gene & $\begin{array}{c}\text { unadjusted } \\
\text { p value }\end{array}$ \\
\hline rs29013431 & 2 & $15,312,400$ & & $5.99 E-03$ \\
rs29012194 & 2 & $26,095,592$ & & $6.28 \mathrm{E}-03$ \\
rs29016537 & 2 & $29,654,789$ & & $1.30 \mathrm{E}-03$ \\
rs29020907 & 4 & $59,252,723$ & IMP2 & $4.97 \mathrm{E}-04$ \\
rs29024570 & 10 & $4,359,586$ & & $4.30 \mathrm{E}-04$ \\
AAFC02107025 & 10 & $21,153,203$ & DHRS1 & $4.73 \mathrm{E}-03$ \\
rs29024728 & 13 & $28,618,368$ & & $3.34 \mathrm{E}-03$ \\
PRNP08 & 13 & $47,214,453$ & PRNP & $4.38 \mathrm{E}-03$ \\
rs29021171 & 14 & $10,106,755$ & & $3.84 \mathrm{E}-03$ \\
SCAFFOLD51887 & 14 & $43,984,153$ & & $7.25 \mathrm{E}-05 *$ \\
rs29014819 & 15 & $35,713,980$ & & $7.17 \mathrm{E}-04$ \\
rS29014820 & 15 & $35,714,095$ & & $6.05 \mathrm{E}-04$ \\
rS29014821 & 15 & $35,714,113$ & & $4.66 \mathrm{E}-04$ \\
AAFC02014662 & 16 & $65,943,749$ & & $7.95 \mathrm{E}-03$ \\
AAFC02012500_1 & 21 & $11,820,092$ & MCTP2 & $1.82 \mathrm{E}-03$ \\
AAFC02012500_2 & 21 & $11,820,201$ & MCTP2 & $3.93 \mathrm{E}-03$ \\
rs29026011 & 21 & $13,254,954$ & LOC618464 & $3.00 \mathrm{E}-03$ \\
rs29019629 & 21 & $33,209,686$ & CSPG4 & $2.01 \mathrm{E}-03$ \\
rs29011202 & 24 & $18,493,192$ & & $9.08 \mathrm{E}-03$ \\
AJ496776 & 28 & $31,495,721$ & & $5.74 \mathrm{E}-03$ \\
\hline Al & & &
\end{tabular}

All locations determined by blast to bovine sequence version 4.0. Threshold of significance is $p \leq 1.7 \times 10^{-5}$ and suggestive significance $p \leq 10^{-4}$ is denoted as *.

known to reside throughout the coding and non-coding regions of PRNP [12]. Of these, 13 PRNP haplotype tagging SNPs (htSNPs) were used in the scans of which 8 were informative for both data sets. The htSNPs used in this study capture a large portion of PRNP haplotype variation observed in a diverse assemblage of U.S cattle, spanning the promoter region into the last exon, however, they do not capture all of it. Additionally, the two InDels previously identified [9-11] as having allele associations with classical BSE were not genotyped in this study and thus no information is available for this sample set and the InDels PRNP haplotypes. One SNP (PRNP08) had a $p$ value of $4.38 \times 10^{-3}$ for an association with classical BSE but did not pass multiple test corrections. Consequently, no significant associations of PRNP variation (the 8 informative htSNPs) with classical BSE were identified in this single marker analysis. Given that the single marker analysis of the informative htSNPs did not capture all of the htSNPs in the PRNP region or the two InDels it should not be considered an exhaustive analysis of the PRNP gene region.

\section{Family-based analysis}

Family-based analyses, although in general being less powerful than case-control studies [19], offer robustness to non-random mating. The transmission/disequilibrium test developed in 1993 by Spielman [17] is intended to test for linkage between complex diseases and genetic markers. The sib-TDT approach used here does not reconstruct parental genotypes in their absence, but uses marker data from unaffected half siblings instead [17]. The DFAM analysis model fits the structure of the related half-sibling sample set and has been utilized in other species as well [20]. The study presented here used a much larger number of markers than the previous studies and used an analysis approach that is robust to population stratification [21]. The advantage this analysis has over the case-control approach is that with 302 affected animals it has twice the number of affected individuals, and thus, a higher study power and likelihood of detecting markers associated with disease loci.

Many of the samples included in the family sample set used in this study were also used by Hernández-Sánchez et al. [13] and Zhang et al. [14], while the case-control sample set was analyzed here for the first time. Hernández-Sánchez et al. [13] also used a TDT approach, however, their analysis method requires heterozygous parents to allow the parent of origin of alleles to be unequivocally determined and as a result many animals had to be disregarded in their analysis. In addition, progeny with the same genotype as the predicted genotype of the sires or progeny that were themselves homozygous were excluded. As a result, although the TDT method used by Hernández- Sánchez et al. [13] has the potential to be powerful, the use of this approach with microsatellite marker based data was limited by the number of genotypes which could be used and ranged from 92 (in the case of marker BMS1658) to 210 (in the case of INRA36). The analysis method used to localize QTLs by Zhang et al. [14] was a regression approach which does not require the parents to be heterozygous and hence all individuals could be included in the analysis. However, the QTL approach is not robust to population stratification. Moreover, the total number of samples (360) used in the Zhang et al. [14] study was smaller than that of Hernández-Sánchez et al. [13] (530) as well as this study (412). The two previous analyses of overlapping family samples yielded different results: the TDT analysis of Hernández-Sánchez et al. [13] found evidence for associations with BSE incidence on chromosomes 5, 10 and 20, whereas the QTL analysis by Zhang et al. [14] identified BSE associated QTL chromosomes 1, 6, 13, 17, 19 and $\mathrm{X} / \mathrm{Y}_{\mathrm{ps}}$. SNPs in the regions matching regions found in previous studies are detailed in Table 3. With regards to the family data this study offers a similar power to that of the TDT analysis by Hernández-Sánchez et al. [13], however uses half-sib controls as opposed to inferring the sires genotype. In addition, due to necessary genotypic restrictions (only 
Table 3 Comparison of SNPs observed in this study with locations indentified in other studies.

\begin{tabular}{|c|c|c|c|c|c|c|}
\hline & & Analysis & & Location & Previously & Peak \\
\hline CHR & SNP ID & Method & p-value & $\mathrm{Mb}$ & Observed & Location Mb \\
\hline 1 & rs29009859 & sib-TDT & $2.0 \times 10^{-5}$ & 89.7 & [14] & 106.5 \\
\hline 5 & AAFC02012009 & sib-TDT & $* * 9.9 \times 10^{-3}$ & 30.6 & No & \\
\hline 5 & E25B16-36408-3 & sib-TDT & $* * 8.8 \times 10^{-5}$ & 66.5 & No & \\
\hline 5 & rs29012226 & sib-TDT & $* * 1.9 \times 10^{-3}$ & 67.7 & No & \\
\hline 5 & rs29024670 & sib-TDT & $3.0 \times 10^{-5}$ & 112.8 & [13] & 107.0 \\
\hline 6 & SCAFFOLD106936_12205 & sib-TDT & $* * 1.8 \times 10^{-3}$ & 98.8 & No & \\
\hline 10 & rs29024570 & Case-control & $* 4.3 \times 10^{-4}$ & 4.4 & No & \\
\hline 10 & rs29013631 & sib-TDT & $* * 6.5 \times 10^{-3}$ & 38.7 & No & \\
\hline 10 & rs29015623 & sib-TDT & $9.0 \times 10^{-5}$ & 48.0 & [13] & 40.0 \\
\hline 17 & rs29021871 & sib-TDT & $* * 3.0 \times 10^{-3}$ & 44.2 & No & \\
\hline 19 & rs29027102 & sib-TDT & $2.0 \times 10^{-5}$ & 62.5 & [14] & 53.5 \\
\hline 20 & rs29018531 & sib-TDT & $* * 3.7 \times 10^{-3}$ & 38.8 & [13] & 46.0 \\
\hline 20 & CART-SNP & sib-TDT & $* * 1.0 \times 10^{-4}$ & $4.9^{\#}$ & No & \\
\hline$x$ & SCAFFOLD68962_9331 & sib-TDT & $* * 1.0 \times 10^{-5}$ & 7.6 & No & \\
\hline$x$ & SCAFFOLD285727_12117 & sib-TDT & $1.0 \times 10^{-5}$ & 85.5 & [14] & 112.5 \\
\hline
\end{tabular}

Sib-TDT $\mathrm{p}$ values are reported as unadjusted unless denoted by ** which indicates Bonferroni corrected values that pass $10,000,000$ permutations. All case control $p$-values are reported are as unadjusted and suggested significance is denoted as *. Only SNPs with appreciable or significant $\mathrm{p}$-values located within the confidence intervals of ref\# [14] or within $10 \mathrm{cM}$ of ref\# [13] are reported here. The location determined by older bovine sequence version 2.0 is denoted by \#. All other locations were determined by blast to bovine sequence version 4.0 and are reported in mega bases. The previous studies reported location in $\mathrm{cM}$ however for the sake of uniformity the location is reported here, based on marker positions, in $\mathrm{Mb}$.

heterozygous genotypes can be used) of the TDT method, the approach used here allowed for a greater number of animals to be included in the analysis. Although the total number of animals used in this analysis includes two smaller families ( 6 families versus 4 families in the previous studies $[13,14])$ this analysis method does not infer sire genotypes and therefore the inclusion of the two smaller families do not reduce the overall power of this analysis.

The sib-TDT analysis in this study identified two significant SNPs on BTA 20 associated with BSE incidence, rs29018531 at $38.8 \mathrm{Mb}$ and a SNP within the cocaine and amphetamine responsive transcript peptide gene, (CART). CART is not currently on the Btau4.0 bovine sequence assembly but was previously mapped to chromosome 20 at $38.5 \mathrm{cR}$ [15]. In addition, the location of CART was reported on Btau2.0 as $4.92 \mathrm{Mb}$ as well as the Maryland map as $9.78 \mathrm{Mb}$. Therefore it is unclear from this study if the observed associations identified here are attributable to one locus or two separate loci. The study of Hernández-Sánchez et al. [13] observed an association with marker INRA36 (at $37.9 \mathrm{Mb}$ ) on BTA 20 with BSE incidence. This study also identified significant markers on chromosomes 5, 6, 10, 17 and X associated with BSE. The study by Hernández-Sánchez et al. [13] also reports associations on BTA 5 and 10 but did not report confidence intervals. The marker identified on BTA 6 in this study (Table 3) is in the same chromosomal region as the marker described by Zhang et al. [14] and is within the confidence interval. The precise location of these significant markers can be found in Table 3. Moreover, the significant marker on BTA 6, Scaffold106936 at $98.7 \mathrm{Mb}$ corresponds with the QTL region, 51-72 $\mathrm{cM}$ on mouse chromosome 5 , previously associated with susceptibility to TSE in mice [22]. Additionally, the homologous region to that identified on BTA 6 was also identified as a QTL modulating scrapie incubation period in sheep [23]. Interestingly, the QTL region on mouse chromosome 5 described by Moreno et al. [22] also corresponds to the location of a significant SNP identified on BTA 17, 44.2 Mb. Thus these chromosomal regions identified in the present study are also supported by studies in cattle and other species. Comparative locations were determined by using the National Center for Biotechnology information map viewer of the mouse QTL regions (build 37.1), then the human and Btrna were selected and bovine locations were determined.

In QTL studies in mice Monero et al., 2008 [22] and Llyod et al., 2002 [24] used a panel of 72 microsatellites to examine $282 \mathrm{~F} 2$ and $124 \mathrm{~F} 2$ mice respectively. However these mice were inoculated intracerebrally as compared to our study where infectious material was orally ingested through contaminated feed. Comparatively these studies have fewer animals and although they use microsatellite markers the information content is limited due to the fact that both studies only use 2 inline bred strains. In addition, it should be noted that single marker association analysis, as reported in this manuscript differs from QTL analysis. These differences make direct 
comparison rather difficult. Given that neither this study nor the previous studies, even in other species, can really be considered comprehensive genome scans they suffer from the same shortfalls. In this study there remains the distinct possibility of a type 2 error which fails to identify a genome location that is associated with disease.

\section{Case-control analysis}

In selecting the analysis approach, it is important to match the appropriate model to the data structure to maximize the power. This study used a case-control approach to analyze the paired control with BSE animals. This approach is powerful in its ability to detect loci linked with disease: however, it has been criticized by geneticists for its lack of robustness for population stratification arising from non-random mating or unknown relationships between individuals [19]. The data presented here was examined for stratification and none was observed (see Additional file 2). However, it is likely that some family relationships exist between some of the cases and controls but the extent and the effect was unable to be determined. Despite the power of the case-control approach, this study was limited by the relatively small number of animals used (149 cases and 184 controls) and no significant results were observed. An increase in the number of cases and controls included in this sample set would have a dramatic effect on the power to detect loci associated with disease [19], as would a higher density scan conducted with an increased number of genome-wide markers.

\section{Shared regions identified in the sib-TDT family and implicated in the case-control analysis}

Many of the SNPs included in the panel are in close proximity and are in LD in Holstein [16]. Therefore, it may be more appropriate to consider the results in terms of chromosomal regions instead of individual markers. Linkage disequilibrium will result in the alleles of several closely spaced SNPs being associated with disease status because they all fall on the same haplotype. Thus, it would be expected that several SNPs in LD with a locus involved in disease would show significant associations. Examples of this can be observed with the loci on BTA 15 in Table 2 as well as BTA 4 in Table 1. In addition, if the same regions give significant or a nearly significant association across the different sample sets, this would also increase confidence that the association is real. Chromosome 2 is a good example: the most significant marker in this study, AAFC02065030, with a Bonferroni corrected $\mathrm{p}=5.5 \times 10^{-5}$ in the family based analysis is at $37.1 \mathrm{Mb}$ on this chromosome. In the case-control samples set, three markers were identified on Chromosome 2, one at $15.3 \mathrm{Mb}$, another at $26.1 \mathrm{Mb}$, and the last $\left(\mathrm{p}=1.0 \times 10^{-3}\right)$ at $29.7 \mathrm{Mb}$. From the different analyses methods and across the two sample sets four markers were identified in the chromosomal region from 15.3 Mb-37.1 Mb on BTA2, with the region of 29$37 \mathrm{Mb}$ being significant. This data supports an association between chromosome 2 and BSE disease, however from the data it cannot be determined if this effect is from one locus or several loci spread across the region.

Another chromosomal region which harbours significant markers that were identified in the family-based analyses and was observed but failed to reach significance in the case-control analysis is on chromosome 21. Four SNPs were observed on BTA 21 from the analysis of case-control samples, two SNPs which are located at 11.8 $\mathrm{Mb}\left(\mathrm{p}=4.4 \times 10^{-3}\right.$ and $\left.\mathrm{p}=8.3 \times 10^{-3}\right)$ in the MCTP2 gene, another at $13.3 \mathrm{Mb}\left(\mathrm{p}=2.5 \times 10^{-3}\right)$ in LOC618464, and an additional SNP at $33.2 \mathrm{Mb}\left(\mathrm{p}=2.0 \times 10^{-3}\right)$ in the gene CSPG4. The family based sib-TDT analysis identified three significant SNPs, at $5.7 \mathrm{Mb}$ in the $M E F 2$ gene $\left(\mathrm{p}=3.5 \times 10^{-4}\right), 12.6 \mathrm{Mb}\left(\mathrm{p}=5.2 \times 10^{-4}\right)$, and $24.83 \mathrm{Mb}$ $\left(\mathrm{p}=5.4 \times 10^{-3}\right)$. When considering both sample sets there appears to be an interval from 11.8 Mb-13.3 Mb with the outer range of approximately 5.7-33.2 Mb for a locus with an effect on BSE. BTA 21 shares conservation of synteny with sheep OAR 16 where QTLs for scrapie susceptibility and scrapie incubation period have been reported [25].

Regions containing loci significantly associated with disease status identified on chromosomes 14,16 and 28 in the sib-TDT analysis of the family-based data were also observed to be of interest, but failed to reach significance, in the case-control study. On chromosome 14 the marker, rs29021171, at $10.1 \mathrm{Mb}$ had a $\mathrm{p}=2.3 \times 10^{-3}$ in the analysis of case-control animals and is in relative close proximity to, rs 29010388 , at $4.2 \mathrm{Mb}$ which was identified as significant in the sib-TDT analysis $(\mathrm{p}=1.2 \times$ $\left.10^{-3}\right)$. On chromosome 16 marker AAFC02014662 at 65.9 $\mathrm{Mb}$ identified in the case-control $\left(\mathrm{p}=7.95 \times 10^{-3}\right)$ is in close proximity to rs29010371 at $63.5 \mathrm{Mb}$, which is in the gene FAM129A, that was identified as significant $(\mathrm{p}=3.0$ $\times 10^{-3}$ ) in the sib-TDT analysis. Finally on chromosome 28 marker AJ496776, at $31.5 \mathrm{Mb}$ identified in the casecontrol sample analysis $\left(\mathrm{p}=9.9 \times 10^{-3}\right)$ is in close proximity to the marker SCAFFOLD176855 (within SLC29A3 gene) at $26.9 \mathrm{Mb}$ identified as significant $\left(\mathrm{p}=2.9 \times 10^{-3}\right)$ in the sib-TDT analysis. None of these chromosomal regions have been previously reported as being associated with BSE.

\section{Candidate genes identified in the case-control and/or sib-TDT family analysis}

The SNPs identified from the family samples on chromosomes 4, 5, 9, 12, 16, 20, 21, 22 and 28 are all found within genes; however, the polymorphisms on 
chromosomes 4 and 12 are in hypothetical genes. The most notable is the polymorphism on chromosome 5 , rs29012226, in the ankyrin repeat and sterile alpha motif domain containing $1 \mathrm{~B}$ gene $(A N K S 1 B)$. This gene is also known as amyloid beta protein precursor $(A P P)$ intracellular domain associated protein 1 (AIDA-1), and is associated with $A P P$ binding [26]. It is well known that APP generates beta amyloid and plays a key role in Alzheimers disease [27-30]. Further cellular prion protein and AIDA-1 has been implicated as a receptor for amyloid- $\beta$ oligomers $[31,26]$, making $A N K S 1 B$ a good candidate gene for further study.

Another candidate gene, which is in close proximity to three SNPs on BTA 2 at $\sim 29.3 \mathrm{Mb}$, with alleles that associated with BSE incidence is B3GALT1. Beta-1,3galactosyltransferase (B3GALT1), is a transferase polypeptide gene involved in the biosynthesis of GPI anchors. The involvement of the GPI anchor, with lipid raft and TSE disease has been investigated [32] and it is thought that the GPI anchor may affect the conformation, or the association of the prion protein with specific membrane domains [33]. An additional potential candidate gene is $C A R T$, cocaine and amphetamine regulate transcript. This neuropeptide plays a role in a variety of physiological processes, some of which include: promotion of hippocampal neurons by upregulating brainderived neurotrophic factors [34] and synaptogenesis [35]. In addition, the expression of CART has shown to be down regulated in mouse prion disease [36].

The SNP located on BTA 14 (43.9 Mb), associated with BSE incidence in the case-control analysis, is in close proximity to the gene exostoses (multiple) 1, EXT1. McCormick et al., [37] showed that EXT1 is an endoplasmic reticulum (ER)-resident type II transmembrane glycoprotein whose expression in cells results in the alteration of the synthesis and display of cell surface heparan sulfate glycosaminoglycans (GAGs). The $\mathrm{N}$ terminus of PrP contains a GAG-binding motif and it is thought that PrP binding of GAG is important in prion disease [37-39]. Additionally, this region contains another candidate gene STMN2, which has been identified in a whole genome association study for genetic risk factors for variant Creutzfeldt-Jakob in humans [40]. Specifically, Mead and others [40] found an association with acquired prion diseases, including vCJD $(\mathrm{p}=5.6 \times 10(-5))$, kuru incubation time $(\mathrm{p}=0.017)$, and resistance to kuru $(\mathrm{p}=2.5 \times 10(-4))$, in a region upstream of STMN2 (the gene that encodes SCG10). Superior cervical ganglion 10, SCG10, is a neuronal growth associated protein and may play a role in neuronal differentiation in modulating membrane interaction with the cytoskeleton during neurite outgrowth. STMN2 is at $39.9 \mathrm{Mb}$ on chromosome 14 in cattle, which is in close proximity to EXT1, making both
STMN2 and EXT1 are functional and positional gene candidates.

\section{Conclusions}

The large number of SNP markers and the two sets of animals used in this study make it the most comprehensive study to date to test genetic loci for an association with classical BSE in European Holstein cattle. The genome-wide scan of half sib families identified an association between the genetic loci on 18 chromosomes with BSE incidence in European Holstein cattle, including a region on BTA 20 associated with BSE incidence that has been reported in previous studies. The identification of markers at or near statistical significance within the same chromosomal regions in both sets of samples provides independent evidence for the association of those regions and the presence of one or more genes within the regions influencing the incidence of BSE in cattle. However, these results need to be confirmed in additional cattle populations or other species. The data in this study can be made available upon request.

It is worth noting that this study identified a large number of associations with classical BSE disease incidence throughout the bovine genome versus one single major locus with a large effect in the bovine genome. This would make it difficult to select cattle that are genetically resistant to classical BSE, however the results give some insight into gene pathways important during disease progression.

\section{Methods \\ Animal information}

This study used two sets of samples from cases and controls, but with different structures. The first sample set consisted of female European Holstein collected in the mid 90's and included 149 BSE case and 184 control animals. The control animals were contemporaries of the BSE cases and collected from the same farms. In addition 15 BSE negative, determined by post-mortem histology, and paired control animals were included in the control set. The second sample set was family based and consisted of 302 BSE affected and 179 unaffected half-sib Holsteins from six sire families. All the BSE affected and unaffected cattle within one family were paternal half sibs from the designated sire but with different dams. No DNA samples were available from any of the sires.

In both sample sets cattle designated as BSE positive were first examined by qualified veterinarians. BSE status was subsequently confirmed post-mortem by histology (by the Veterinary Laboratories Agency, New Haw, Surrey, UK). None of the control animals exhibited any clinical symptoms of disease and were presumed to be free of disease. All of the control animals were age and 
sex matched from the same calving season and from the same farm as the BSE cases. As such, the control animals are assumed to have been exposed to the same environment.

\section{DNA isolation and genotyping}

Genomic DNA from the case-control was isolated from blood using a high salt phenol/chloroform extraction method as described by Sherman et al. [41]. Genomic DNA from the family animal set was isolated from blood samples by phenol and chloroform extraction, as described by Hernández-Sánchez et al. [13].

The genotyping panel was comprised of two oligonucleotide pool assays (OPAs) as described by McKay and others in 2007 [16]. Briefly, 5,500 SNP were mapped on the Roslin-Cambridge 3,000 rad bovine-hamster whole genome radiation hybrid panel (WGRH3000) [42] and the minor allele frequency (MAF) was determined on a variety of breeds, including Holstein. Of the original SNPs, 3,072 were selected to give the greatest genome coverage and MAF $>0.05$ for the genome scan. An Illumina GoldenGate assay [43] was performed using the two custom OPAs and genotypes determined using an Illumina BeadScan (Illumina Inc., San Diego, CA) and the Illumina BeadStudio software. Sequences containing SNPs (see additional file 3) were blasted on the bovine assembly (4.0) to determine the SNP locations [16] and, the location for all except 111 SNPs were determined.

\section{Data quality control}

Quality control analyses were carried out by removing confounding effects prior to the data analysis. For the case-control sample set from the original 3,072 SNPs in the assay, 122 SNPs were excluded based on assay failure, as observed by poor clustering of alleles using the BeadStudio software. The remaining 2,950 SNPs were submitted to the PLINK program [18] where seven duplicated SNPs were removed and 12 SNPs were removed because more than $10 \%$ of the samples failed to genotype at that locus (GENO >0.1). Additionally, 13 samples were removed due to low genotyping rate $(\mathrm{MIND}>0.1)$. A further $61 \mathrm{SNPs}$ were removed due to a MAF $<0.01$. The MAFs for each of the SNPs in this data set is provided in additional file 3 and the graphical representation of the MAF for each chromosome is shown in additional file 4 . The remaining individuals that were included in the analyses had a mean genotyping rate of 0.995. Population stratification was tested for in this data set and none was observed (data in additional file 2).

For the family sample set, transmission disequilibrium analysis was performed using the sib-TDT application in
Table 4 The sire identities and the number of half sib offspring analyzed in this study verses the number of animal used two previous studies.

\begin{tabular}{lcccccc}
\hline Sire & \multicolumn{3}{c}{ Number of BSE } & \multicolumn{3}{c}{ Number of control } \\
\hline & This study & {$[13]$} & {$[14]$} & This study & {$[13]$} & {$[14]$} \\
\hline Barold Rock Seal & 33 & 53 & 43 & 11 & 28 & 22 \\
Brynhyfryd Cascade & 68 & 93 & 72 & 28 & 56 & 18 \\
Leighton Workboy & 71 & 88 & 70 & 40 & 44 & 22 \\
Maybar Juniper & 90 & 124 & 83 & 51 & 44 & 30 \\
Bowerchalk Polacca & 4 & 0 & 0 & 6 & 0 & 0 \\
Deri cascade & 3 & 0 & 0 & 7 & 0 & 0 \\
\hline Total & 269 & 358 & 268 & 143 & 172 & 92 \\
\hline
\end{tabular}

the PLINK program. Following the removal of SNPs that did not cluster well, 2,904 SNPs were used in the analysis. Of these loci, a further 22 SNPs were removed due to missing data $(\mathrm{GENO}>0.1)$, seven duplicated SNPs, and a further 48 SNPs were removed due to low allele frequency $(\mathrm{MAF}<0.01)$. The MAFs for each of the SNPs in this data set is provided in additional file 3 and the graphical representation of the MAF for each chromosome is shown as in additional file 4. Of the individuals examined, 5 samples failed and 64 were removed because of low genotyping frequency (MIND>0.1), for the 412 remaining individuals (see Table 4) a genotyping success rate of 0.98 was obtained.

\section{Statistical analysis}

The PLINK software v1.04 was used to perform the majority of the statistical analysis [18]. The data from the case-control sample set were analyzed using the basic case-control association $\left(\chi^{2}\right)$ test. Whereas the family sample set was analyzed with the DFAM program [18], which is an adjusted family TDT analysis, as described by Spielman [17]. To correct for multiple tests Bonferroni single-step adjusted p-values (BONF) procedures were applied. A permutation test was also used in this study, which was $\max (\mathrm{T})$ " permutation with 10,000 and 10,000,000 permutations, a procedure that permutes both a point-wise SNP significance and a genome-wide significance.

Additional file 1: The best fit model analysis results (Genotypic, Recessive, and Dominant) of the case-control sample set.

Additional file 2: Multi-dimensional Scaling (MDS) of the multiple family sample set and unrelated sample set.

Additional file 3: Sequence information for the SNPs and the minor allele frequencies for the family and case-control sample sets in order of position.

Additional file 4: Graphical plots of the MAF versus position for each chromosome for the multiple family sample set and unrelated sample set. 


\section{Abbreviations}

BSE: bovine spongiform encephalopathy; CM: centimorgans; CR: centirad; ER: endoplasmic reticulum; GAG: glycosaminoglycans; GPI: glycosylphosphatidylinositol; htSNP: haplotype tagging single nucleotide polymorphism; InDel: insertion/deletion polymorphism; LD: Linkage disequilibrium; MAF: minor allele frequency; OPA: oligonucleotide pool assay; $\operatorname{PrP}^{C}$ : native prion protein; PrPRes: misfolded prion protein; QTL: quantitative trait loci; Sib-TDT: sibling transmission disequilibrium test; SNP: single nucleotide polymorphism; TSE: transmissible spongiform encephalopathy.

\section{Acknowledgements}

The authors wish to thank Alberta Bovine Genomics group and PrioNet Canada and Alberta Prion Research Institute (200500696) for their financial support. JLW acknowledges the Cariplo Foundation for their support.

\section{Author details}

'Department of Agricultural, Food and Nutritional Science, University of Alberta, Edmonton, Canada. ${ }^{2}$ USDA, ARS, US Meat Animal Research Center, Clay Center, Nebraska, USA. ${ }^{3}$ Department of Veterinary Pathobiology, University of Illinois, Urbana, Illinois, USA. ${ }^{4}$ Department of Animal Science, Washington State University, Pullman, Washington, USA. ${ }^{5}$ Divisions of Animal Sciences, University of Missouri, Columbia, Missouri, USA. 'Parco Tecnologico Padano, Via Einstein, Polo Universitario, Lodi, 26900 Italy.

\section{Authors' contributions}

BMM, SSM carried out the DNA isolation and genotyping and JLW designed strategies for sampling infected and control animals and provided the samples. BMM, MS, PS, AP and ZW participated in the bioinformatics and statistical analysis. BMM, SSM, JLW, MLC, and WWL participated in the study design and helped with the draft of the manuscript. All authors read and approved the final manuscript.

\section{Received: 1 June 2009 Accepted: 29 March 2010}

Published: 29 March 2010

\section{References}

1. Prusiner SB, Hsiao KK: Human prion diseases. Ann Neuro 1994, 35:385-395.

2. Pan KM, Baldwin M, Nguyen J, Gasset M, Serban A, Groth D, Mehlhorn I, Huang Z, Fletterick RJ, Cohen FE, Prusiner SB: Conversion of $\alpha$-helices into $\beta$-sheets features in the formation of the scrapie prion proteins. Proc Natl Acad Sci USA 1993, 90:10962-10966.

3. Cronier S, Gros N, Tattum MH, Jackson GS, Clarke AR, Collinge J, Wadsworth JDF: Detection and characterization of proteinase K-sensitive disease-related prion protein with thermolysin. Biochem J 2008, 416(2):297-305.

4. Atarashi R, Moore RA, Sim VL, Hughson AG, Dorward DW, Onwubiko HA, Priola SA, Caughey B: Ultrasensitive detection of scrapie prion protein using seeded conversion of recombinant prion protein. Nat Methods 2007, 8:645-650.

5. Laegreid WW, Clawson ML, Heaton MP, Green BT, O'Rourke KI, Knowles DP: Scrapie resistance in ARQ sheep. J Virol 2008, 82(20):10318-10320.

6. Alvarez L, Arranz JJ, San Primitivo F: Identification of a new leucine haplotype (ALQ) at codon 154 in the ovine prion protein gene in Spanish sheep. J Anim Sci 2006, 84(2):259-265.

7. Collinge J, Sidle KCL, Meads J, Ironside J, Hill AF: Molecular analysis of prion strain variation and the aetiology of 'new variant' CJD. Nature 1996, 383:685-690

8. Gambetti P, Kong Q, Zou W, Parchi P, Chen SG: Sporadic and familial CJD: classification and characterisation. Br Med Bull 2003, 66:213-239.

9. Sander P, Hamann H, Drögemüller C, Kashkevich K, Schiebel K, Leeb T: Bovine prion protein gene (PRNP) promoter polymorphisms modulate PRNP expression and may be responsible for differences in bovine spongiform encephalopathy susceptibility. J Biol Chem 2005, 280(45):37408-37414

10. Juling K, Schwarzenbacher $H$, Williams JL, Fries R: A major genetic component of BSE susceptibility. BMC Biol 2006, 4:33.

11. Haase B, Doherr MG, Seuberlich T, Drögemüller C, Dolf G, Nicken $P$, Schiebel K, Ziegler U, Groschup MH, Zurbriggen A, Leeb T: PRNP promoter polymorphisms are associated with BSE susceptibility in Swiss and German cattle. BMC Genetics 2007, 8:15.
12. Clawson ML, Heaton MP, Keele JW, Smith TPL, Harhay GP, Laegreid WW: Prion gene haplotypes of U.S. cattle. BMC Genetics 2006, 7:51.

13. Hernández-Sánchez J, Waddington D, Wiener P, Haley CS, Williams JL: Genome-wide search for markers associated with bovine spongiform encephalopathy. Mamm Genome 2002, 13(3):164-168.

14. Zhang C, de Koning DJ, Hernández-Sánchez J, Haley CS, Williams JL, Wiener P: Mapping of multiple quantitative trait loci affecting bovine spongiform encephalopathy. Genetics 2004, 167(4):1863-1872.

15. Anderson RM, Donnelly CA, Ferguson NM, Woolhouse MEJ, Watt CJ, Udy HJ, MaWhinney S, Dunstan SP, Southwood TRE, Wilesmith JW, Ryan JB, Hoinville $L$, Hillerton JE, Austin AR, Wells GA: Transmission dynamics and epidemiology of BSE in British cattle. Nature 1996, 382:779-788.

16. McKay SD, Schnabel RD, Murdoch BM, Aerts J, Gill CA, Gao C, Li C, Matukumalli LK, Stothard P, Wang Z, Van Tassell CP, Williams JL, Taylor JF, Moore SS: Construction of bovine whole-genome radiation hybrid and linkage maps using high-throughput genotyping. Anim Genet 2007, 38(2):120-125.

17. Spielman RS, Ewens WJ: A sibship test for linkage in the presence of association: the sib transmission/disequilibrium test. Am J Hum Genet 1998, 62:450-458.

18. Purcell $S$, Neale B, Todd-Brown K, Thomas L, Ferreira MAR, Bender D, Maller J, Sklar P, de Bakker PIW, Daly MJ, Sham PC: PLINK: A tool set for whole-genome association and population-based linkage analyses. Am J Hum Genet 2007, 81:559-575.

19. Risch N, Teng J: The relative power of family-based and case-control designs for linkage disequilibrium studies of complex human diseases I. DNA pooling. Genome Res 1998, 8:1273-1288.

20. Wiiki A, Wade C, Biagi T, Ropstad E, Bjerkas E, Lindblad-Toh K, Lingaas F: A deletion in Nephronophthisis 4 (NPHP4) is associated with recessive cone-rod dystrophy in standard wire-haired dachshund. Genome research 2008, 18(9):1415-1421.

21. Devlin B, Roeder K, Bacanu SA: Unbiased methods for population-based association studies. Genet Epidem 2001, 21:273-284.

22. Moreno CR, Lantier F, Lantier I, Sarradin P, Elsen JM: Detection of new quantitative trait Loci for susceptibility to transmissible spongiform encephalopathies in mice. Genetics 2003, 165(4):2085-91.

23. Moreno CR, Cosseddu GM, Schibler L, Roig A, Moazami-Goudarzi K, Andreoletti O, Eychenne F, Lajous D, Schelcher F, Cribiu EP, Laurent $P$, Vaiman $D$, Elsen JM: Identification of new quantitative trait loci (other than the PRNP gene) modulating the scrapie incubation period in sheep. Genetics 2008, 179(1):723-726.

24. Lloyd SE, Uphill JB, Targonski PV, Fisher EM, Collinge J: Identification of genetic loci affecting mouse-adapted bovine spongiform encephalopathy incubation time in mice. Nuerogenetics 2002, 4(2):77-81.

25. Laurent $P$, Schibler L, Vaiman A, Laubier J, Delcros C, Cosseddu G, Vaiman D, Cribiu EP, Yerle M: A 12 000-rad whole-genome radiation hybrid panel in sheep: application to the study of the ovine chromosome 18 region containing a QTL for scrapie susceptibility. Anim Genet 2007, 38(4):358-363.

26. Ghersi E, Noviello C, D'Adamio L: Amyloid - $\beta$ protein precursor (A $\beta P P$ ) intracellular domain-associated protein-1 proteins bind to A $\mathrm{PPP}$ and modulate its processing in an isoform-specific manner. JBC 1990, 279(47):49105-49112.

27. Ring S, Weyer SW, Kilian SB, Waldron E, Pietrzik CU, Filippov MA, Herms J, Buchholz C, Eckman CB, Korte M, Wolfer DP, Müller UC: The secreted $\beta$ amyloid precursor protein ectodomain APPs $\alpha$ is sufficient to rescue the anatomical, behavioral, and electrophysiological abnormalities of APPdeficient mice. J Neurosci 2007, 27(29):7817-7826.

28. Li ZW, Stark G, Götz J, Rülicke T, Gschwind M, Huber G, Müller U, Weissmann C: Generation of mice with a 200-kb amyloid precursor protein gene deletion by Cre recombinase-mediated site-specific recombination in embryonic stem cells. Proc Natl Acad Sci USA 1996, 93(12):6158-6162.

29. Müller U, Cristina N, Li ZW, Wolfer DP, Lipp HP, Rülicke T, Brandner S, Aguzzi A, Weissmann C: Behavioral and anatomical deficits in mice homozygous for a modified $\beta$-amyloid precursor protein gene. Cell 1994, 79(5):755-765.

30. Zheng $H$, Jiang M, Trumbauer ME, Sirinathsinghiji DJS, Hopkins R, Smith DW Heavens RP, Dawson GR, Boyce S, Conner MW, Stevens KA, Slunt HH, Sisoda SS, Chen HY, Ploeg Van der LHT: $\beta$-amyloid precursor 
protein-deficient mice show reactive gliosis and decreased locomotor activity. Cell 1995, 81(4):525-531.

31. Laurén J, Gimbel DA, Nygaard HB, Gilbert JW, Strittmatter SM: Cellular prion protein mediates impairment of synaptic plasticity by amyloid- $\beta$ oligomers. Nature 2009, 457(7233):1128-1132.

32. Paulick MG, Bertozzi CR: The glycosylphosphatidylinositol anchor: a complex membrane-anchoring structure for proteins. Biochemistry 2008 , 47(27):6991-7000.

33. Campana V, Caputo A, Sarnataro D, Paladino S, Tivodar S, Zurzolo C: Characterization of the properties and trafficking of an anchorless form of the prion protein. J Biol Chem 2007, 282(31):22747-22756.

34. Wu B, Hu S, Yang M, Pan H, Zhu S: CART peptide promotes the survival of hippocampal neurons by upregulating brain-derived neurotrophic factor. Biochem Biophys Res Commun 2006, 347(3):656-661.

35. Abrahám H, Orsi G, Seress L: Ontogeny of cocaine- and amphetamineregulated transcript (CART) peptide and calbindin immunoreactivity in granule cells of the dentate gyrus in the rat. Int I Dev Neurosci 2007 25(5):265-274

36. Sorensen G, Medina S, Parchaliuk D, Phillipson C, Robertson C, Booth SA: Comprehensive transcriptional profiling of prion infection in mouse models reveals networks of responsive genes. BMC Genomics 2008, 9:114.

37. McCormick C, Leduc Y, Martindale D, Mattison K, Esford LE, Dyer AP, Tufaro F: The putative tumour suppressor EXT1 alters the expression of cell-surface heparan sulfate. Nat Genet 1998, 19(2):158-161.

38. Yin S, Pham N, Yu S, Li C, Wong P, Chang B, Kang SC, Biasini E, Tien P, Harris DA, Sy MS: Human prion proteins with pathogenic mutations share common conformational changes resulting in enhanced binding to glycosaminoglycans. Proc Natl Acad Sci USA 2007, 104(18):7546-7551.

39. Yu S, Yin S, Pham N, Wong P, Kang SC, Petersen RB, Li C, Sy MS: Ligand binding promotes prion protein aggregation-role of the octapeptide repeats. FEBS J 2008, 275(22):5564-5575.

40. Mead S, Poulter M, Uphill J, Beck J, Whitfield J, Webb TEF, Campbell T, Adamson G, Deriziotis P, Tabrizi SJ, Hummerich H, Verzilli C, Alpers MP, Whittaker JC, Collinge J: Genetic risk factors for variant Creutzfeldt-Jakob disease: a genome-wide association study. Lancet Neurol 2009, 8(1):57-66

41. Sherman EL, Nkrumah DJ, Murdoch BM, Moore SS: Identification of polymorphisms influencing feed intake and efficiency in beef cattle. Anim Genet 2008, 39(3):225-231.

42. Williams JL, Eggen A, Ferretti L, Farr CJ, Gautier M, Amati G, Ball G, Caramorr T, Critcher R, Costa $S$, Hextall P, Hills D, Jeulin A, Kiguwa $S$, Ross $O$ Smith AL, Saunier K, Urquhart B, Waddington D: A bovine whole-genome radiation hybrid panel and outline map. Mamm Genome 2002, 13(8):469-474.

43. Oliphant A, Barker DL, Stuelpnagel JR, Chee MS: BeadArray technology: enabling an accurate, cost-effective approach to high-throughput genotyping. Biotechniques 2002, Suppl 56-8: 60-61.

doi:10.1186/1471-2156-11-20

Cite this article as: Murdoch et al: A 2cM genome-wide scan of European Holstein cattle affected by classical BSE. BMC Genetics 2010 11:20.

\section{Submit your next manuscript to BioMed Central and take full advantage of:}

- Convenient online submission

- Thorough peer review

- No space constraints or color figure charges

- Immediate publication on acceptance

- Inclusion in PubMed, CAS, Scopus and Google Scholar

- Research which is freely available for redistribution

Submit your manuscript at www.biomedcentral.com/submit
Biomed Central 